\title{
Intestinal obstruction induced by phytobezoars
}

\author{
Jia-Hong Chen MD, Kuan-Yu Chen MD, Wei-Kuo Chang MD PhD
}

Previously published at www.cmaj.ca

A 77-year-old man was admitted because of periumbilical pain, vomiting, abdominal distension and no passage of stool for four days. These symptoms were resistant to the treatment of oral magnesium oxide and glycerin suppositories given at an outpatient clinic. The patient's surgical history consisted of a cholecystectomy 10 years before admission and an appendectomy at the age of 70 years. He was receiving treatment for hypertension and type 2 diabetes mellitus.

On examination, the patient had increased bowel sounds and epigastric tenderness without rebound pain. No palpable mass was found on rectal examination. Initial laboratory results showed elevated blood urea nitrogen at 20 (normal 2.5-10.7) $\mathrm{mmol} / \mathrm{L}$, but were otherwise normal. A plain radiograph of the abdomen was consistent with gas ileus and a nasogastric tube was inserted. Some foul loose stool-like material was noted to be draining from the nasogastric tube.

Computed tomographic (CT) scans of the abdomen showed an intraluminal inhomogeneous mass $(5.9 \mathrm{~cm}$ in diameter) with mottled gas pattern within it over the splenic flexure of the descending colon, and another bezoar-like opacity over the distal jejunum, with obstruction of the proximal colon and small intestine (Figure 1). No adhesive bands were visible.

Colonoscopy was performed for further diagnosis but was nondiagnostic because of the presence of a large amount of stool. Based on the radiologic findings, a clinical diagnosis of bezoars causing intestinal obstruction was made. It was unclear whether possible adhesions from the previous surgery may have contributed to the formation of the bezoars.

After a careful dietary history was taken, we found that the patient had eaten an orange per day for several years and had recently increased his intake of oranges. Because he was edentulous, he often swallowed the orange sections without chewing them. After treatment with nasogastric tube decompression and nothing by mouth, the patient's symptoms improved

From the Division of Gastroenterology (K.-Y. Chen, Chang) and the Department of Medicine (J.-H. Chen), Tri-Service General Hospital, Nationa Defense Medical Center, Taipei, Taiwan

CMAJ 2010. DOI:10.1503/cmaj.090316 progressively. The patient was discharged in stable condition.

Phytobezoars are concretions of poorly digested fruit and vegetable fibres that may develop in the gastrointestinal tract, and account for $0.4 \%-4 \%$ of all intestinal obstructions. ${ }^{1}$ Phytobezoars related to intake of oranges are more common in patients with a history of abdominal surgery. ${ }^{2}$ The characteristic CT findings alerted us to the diagnosis of a bezoar-induced intestinal obstruction. Although in our patient a nasogastric tube for decompression and conservative treatment improved the symptoms without surgery, some patients may require surgery. ${ }^{1,3,4}$ Patients with a history of bezoar formation may want to avoid excessive intake of foods rich in cellulose, such as oranges, persimmons and celery.

This article has been peer reviewed.

Competing interests: None declared.

\section{REFERENCES}

1. Teng HC, Nawawi O, Ng KL, et al. Phytobezoar: an unusual cause of intestinal obstruction. Biomed Imaging Interv J 2005; 1:4

2. Acar T, Tuncal S, Aydin R. An unusual cause of gastrointestinal obstruction: bezoar. NZ Med J 2003;116:U422.

3. Rubin M, Shiminov M, Drief F, et al. Phytobezoar: a rare cause of intestinal obstruction. Dig Surg 1998;15:52-4.

4. Retter J, Neff W, Singer MV. Small bowel obstruction produced by a phytobezoar. Clin Gastroenterol Hepatol 2008;6:A20. 